\title{
Some Aspects of Probability and Possi- bility Theories for Numerical Analysis of Uncertain Mechanical Systems
}

\author{
Milan Sága (SK) milan.saga@fstroj.uniza.sk \\ Vladimír Dekýš (SK) vladimir.dekys@fstroj.uniza.sk \\ Milan Vaško (SK) milan.vasko@fstroj.uniza.sk
}

\begin{abstract}
BIOGRAPHICAL NOTES
Milan Sága, prof. Dr. Ing., works as a university teacher at the University of Žilina in Žilina. He was born in Žilina in 1965. In 1989 he finished his university studies at the Faculty of Mechanical and Electrical Engineering at the University of Transport and Communications in Žilina, specialization Transport and Manipulation Technology. In 1995 he defended the dissertation with the title Optimum Design of Chosen Parameters of Track Maintenance Machines and he acquired a research degree Doctor in the scientific field Transport and Manipulation Technology at the Faculty of Mechanical Engineering of the University of Transport and Communications in Žilina. In 1999 he habilitated for an associate professor with the title of his work Analysis and Synthesis of Nonlinear Mechanical Systems with Random Inputs in the scientific field Applied Mechanics at the Faculty of Mechanical Engineering of the University of Žilina. In 2006 he was inaugurated for a professor in the scientific field Machine elements and mechanisms at the Faculty of Mechanical Engineering of the University of Žilina. Since 2004 he has been the vicedean of the Faculty of Mechanical Engineering of the University of Žilina and since 2005 as a head of the Department of Applied Mechanics. In his scientific, research and pedagogical activities, he deals mainly with mechanics of bodies, computer optimization of structures, multiaxial fatigue, life prediction, stochastic vibration and fuzzy analysis of mechanical systems with uncertain parameters. He is author and co-author of more than 145 scientific papers, 8 textbooks and 5 monographs.
\end{abstract}

Vladimír Dekýš, doc. Ing. CSc., works as a university teacher at the University of Žilina. He was born in Zvolen in 1957. In 1981 he finished his university studies at the Faculty of Nuclear Sciences and Physical Engineering of the Czech Technical University (CVUT) in Prague, specialization Physical Engineering. He worked in Slovak Academie of Science in Martin (reliability of mechanical systems and accelerated tests), ZTS Research and Development Institute in Martin - ZTS VVU Martin (reliability, test of agricultural tractors, gears and hydraulics) and condition monitoring. He acquired a research degree CSc. (PhD.) in the scientific field Dynamics of Rigid and Flexible Bodies at the Slovac Academy of Science in Bratislava. He worked at the The Department of Applied Mechanics of Faculty of Mechanical Engineering, University of Žilina since 2001. In 2007 he habilitated for an associate professor with the title of his work Valuation of Machine Equipments by using Vibration Measurements. In his scientific, research and pedagogical activities, he deals mainly with dynamics, reliability, experimental mechanics, modal analysis and condition 
monitoring.

Milan Vaško, Ing. PhD., he was born in Poprad in 1971. He works as the university teacher at the University of Žilina in Žilina. In 1998 he finished his university studies at the Faculty of Mechanical Engineering at the University of Transport and Communications in Žilina. In 2007 he defended the dissertation and he acquired a research degree PhD in the scientific field Applied Mechanics at the Faculty of Mechanical Engineering of the University of Žilina. Since 2009 he become head of the Sub-department of Elasticity and Strength in the scientific field Applied Mechanics. In his scientific, research and pedagogical activities he deals mainly with computer optimization of structures, life prediction and analysis of mechanical systems with uncertain parameters. $\mathrm{He}$ is author and co-author of more than 70 technical papers published in domestic and foreign scientific journals and anthologies and 3 lecture notes and 2 monographs.

\section{KEY WORDS}

Uncertain Parameter, Optimization, Interval Number, Interval Arithmetic, Fuzzy Set, Random Variable, INTLAB, Monte Carlo Method

\section{ABSTRACT}

Our paper presents chosen traditional (based on probability theory) and non-traditional (based on possibility theory) computational tools for analysis of the material, geometric or loading uncertainties in mechanical structures. Uncertainties are introduced as bounded possible values - intervals or as fuzzy sets, assuming possibility theory and as random parameters in the case of the probability theory. The main goal is to propose numerical algorithms for interval modal and spectral FE computations suggested by authors and their additional implementation into fuzzy analysis and Monte Carlo method.

\section{INTRODUCTION}

In the last decade there has been an increased interest in the modeling and analysis of engineering systems under uncertainties. To obtain reliable results for the solutions of engineering problems, exact values for the parameters of the model equations should be available. In the reality, however, those values often can not be provided, and the models usually show a rather high degree of un- certainty. Computational mechanics, for example, encoun-ters uncertainties in geometric, material and load parameters as well as in the model itself and in the analysis procedure too. For that reason, the responses, such as displacements, stresses, natural frequency, or other dynamic characteristics, will usually show some degree of uncertainty [8]. It means that the obtained result using one specific value as the most significant value for an uncertain parameter cannot be considered as representative for the whole spectrum of possible results.

It is generally known that probabilistic modeling and statistical analysis are well established for modeling of mechanical systems with uncertainties. In addition, a number of non-probabilistic computational techniques have been proposed, e.g. fuzzy set theory [1, 8], interval approach [1, 3, 5, 6, 7], imprecise probabilities [3] etc. The growing interest in these approaches originated from a criticism of the credibility of probabilistic approach when input dates are insufficient. It is argued that the new non-probabilistic treatments could be more appropriate in the modeling of the vagueness.

\section{METHODS OF INTERVAL AND FUZZY ANALYSIS Interval Analysis}

Interval arithmetic was developed by Moore [6] while studying the propagation and control of truncation and rounding off the error, using floating point arithmetic on a digital computer. Moore was able to generalize this work into the arithmetic independence of machine considera-tions. In this approach, an uncertain number is represented by an interval of real numbers. The interval numbers derived from the experimental data or expert knowledge can then take into account the uncertainties in the model parameters, model inputs etc. By this technique, the complete information about the uncertainties in the model may be included and one can demonstrate how these uncertainties are processed by the calculation procedure INTLAB implemented in MATLAB [2].

The alternative avenue of the interval arithmetic is to use the Monte Carlo technique (MC) [8]. With the advent of recent computa-tional facilities, this method becomes attractive. The results are determined from the series of numerical analyses (approximately 1000 to 10000 iterations). It is recommended to generate the random values with the uniform distribution. 
The comparison has been realized with the usage of midpoint residual vector $\mathbf{r}_{\text {Midpoint }}$ and radius residual vector $\mathbf{r}_{\text {Residual }}$ expressed in \%, e.g.

$$
\begin{aligned}
& \mathbf{r}_{\text {Midpo int }}=\left|\frac{\operatorname{mid}\left(\mathbf{y}_{\text {Intlab }}\right)-\operatorname{mid}\left(\mathbf{y}_{\text {MC }}\right)}{\operatorname{mid}\left(\mathbf{y}_{\text {Intlab }}\right)}\right| \cdot 100 \% \\
& \mathbf{r}_{\text {Radius }}=\left|\frac{\operatorname{rad}\left(\mathbf{y}_{\text {Intlab }}\right)-\operatorname{rad}\left(\mathbf{y}_{\text {MC }}\right)}{\operatorname{rad}\left(\mathbf{y}_{\text {Intlab }}\right)}\right| \cdot 100 \%
\end{aligned}
$$

During the solving of the particular tasks in the engineering practice using the interval arithmetic application on the solution of numerical mathematics and mechanical problems, the problem known as the overestimate effect is encountered. Its elimination is possible only in the case of meeting the specific assumptions, mainly related to the time efficiency of the computing procedures. Now, we will try to analyze some solution approaches already used or proposed by the authors. We will consider the following methods:

\section{- Monte Carlo method (MC),}

method of a solution evaluation in marginal values of interval parameters - infimum and supremum (COM1),

\section{method of a solution evaluation for all marginal values of interval parameters - all combinations of infimum and supremum (COM2), \\ method of infimum and supremum searching us- ing some optimizing technique application (OPT), \\ direct application of the interval arithmetic us- ing INTLAB MATLAB's toolbox (INTL).}

Monte Carlo method (MC) is a time consuming but reliable solution. Various combinations of the uncertain parameter deterministic values are generated and after the subsequent solution in the deterministic sense we obtain a complete set of results processed in an appropriate manner. Infimum and supremum calculation is following

$\inf (F)=$ min of all results of $F\left(p_{i}\right)$

$\sup (F)=$ max of all results of $F\left(p_{i}\right)$

where $i=1, \ldots, \mathrm{m}$ and $\approx \mathrm{m} 5000 \div 10000$.

Second method application (COM1), i.e. solution evaluation in marginal values of interval parameters has its physical meaning for many engineering problems. We consider an approach where the extreme output values are obtained by the application of the extreme parameter values on input.
That means that the infimum/supremum is obtained using the deterministic analysis for infimum or supremum of input uncertain parameters. Infsup calculation is

$$
\begin{aligned}
& \inf (F)=\min \text { of }[F(\underline{p}), F(\bar{p})] \\
& \sup (F)=\max \text { of }[F(\underline{p}), F(\bar{p})]
\end{aligned}
$$

The third approach (COM2) which is also based on the set of the deterministic analyses appears as the more suitable one. The marginal interval parameter values are considered again but the infimums and supremums are also combined. The method provides satisfying results and can be marked as reliable, even if there is still a doubt about the existence of the extreme solution for the uncertain parameter inner values. Solution for two interval numbers $p_{1}=<a_{1} \quad b_{1}>$ and $p_{2}=<a_{2} \quad b_{2}>$ may be found by this computational way

$$
\begin{aligned}
& \inf (F)= \\
& =\min \text { of }\left[F\left(a_{1} a_{2}\right), F\left(a_{1} b_{2}\right), F\left(b_{1} a_{2}\right), F\left(b_{1} b_{2}\right)\right], \\
& \sup (F)= \\
& =\max \text { of }\left[F\left(a_{1} a_{2}\right), F\left(a_{1} b_{2}\right), F\left(b_{1} a_{2}\right), F\left(b_{1} b_{2}\right)\right] .
\end{aligned}
$$

The method of the infimum and supremum solution searching using the optimization techniques (OPT) is proposed by the authors as an alternative to the first and to the third method. It should eliminate a big amount of analyses in the first method and also eliminates the problem with the possibility of the infimum and supremum existence inside of the interval parameters for the deterministic values. Computational process for two interval numbers $\left.\left.p_{1}=<a_{1} b_{1}\right\rangle, p_{2}=<a_{2} b_{2}\right\rangle$ may be found as follows

$\inf (F)=F\left(\mathbf{p}_{\text {OPT }}\right)$,

i.e. find $\mathbf{p}_{O P T}$ that $F\left(\mathbf{p}_{O P T}\right) \rightarrow \min$,

$\sup (F)=F\left(\mathbf{p}_{O P T}\right)$,

i.e. find $\mathbf{p}_{O P T}$ that $F\left(\mathbf{p}_{O P T}\right) \rightarrow \max$.

The authors used also the interval arithmetic principles implemented in INTLAB as another computing tool. However, the overestimate effect mentioned above for the significant uncertainties causes considerable problems and the possibilities of INTLAB using are therefore very restricted. 
INTLAB using makes sense particularly for simple problems because of the results obtaining speed. Interval Eigenvalues Finite Elements Analysis (IFEA) The finite element method (FEM) $[3,5,8]$ is a very popular tool for a complicated structural analysis. The ability to predict the behavior of a structure under static or dynamic loads is not only of a great scientific value, it is also very useful from an economical point of view. A reliable FE analysis could reduce the need for prototype production and therefore significantly reduce the associated design validation cost.

It is sometimes very difficult to define a reliable FE model for realistic mechanical structures when a number of its physical properties is uncertain. Particularly, in the case of FE analysis, the mechanical properties of the used materials are very hard to predict, and therefore an important source of uncertainty. Reliable vali-dation can only be based on an analysis which takes into account all uncertainties that could cause this variability. It is the aim of this part to incorporate the most important uncertainties in FE analysis.

According to the character of the uncertainty, we can define a structural uncertainty (geometrical and material parameters) and uncertainty in load (external forces, etc.). The structural uncertainty parameters are usually written into vector $\mathbf{x}=[\underline{\mathbf{x}}, \overline{\mathbf{x}}]$ and the interval modal FE analysis may be formulated as follows

$$
\begin{aligned}
& {\left[\mathbf{K}(\mathbf{x})-\lambda_{j} \cdot \mathbf{M}(\mathbf{x})\right] \cdot \mathbf{v}_{j}=\mathbf{0}} \\
& \left([\underline{\mathbf{K}}, \overline{\mathbf{K}}]-\left[\underline{\lambda_{\mathrm{j}}}, \overline{\lambda_{\mathrm{j}}}\right] \cdot[\underline{\mathbf{M}}, \overline{\mathbf{M}}]\right) \cdot\left[\underline{\mathbf{v}_{j}}, \overline{\mathbf{v}_{j}}\right]=\mathbf{0}
\end{aligned}
$$

where $\lambda_{\mathrm{j}}, \overline{\lambda_{\mathrm{j}}}$ and $\mathbf{v}_{j}, \overline{\mathbf{v}_{j}}$ are the $j$-th eigenvalue with corresponding eigenvector, $\underline{\mathbf{K}}, \overline{\mathbf{K}}, \underline{\mathbf{M}}, \overline{\mathbf{M}}$ are of course the infimum and supremum of the mass and stiffness matrices. The application of the classic interval arithmetic for FE analysis is very limited. Its overestimation grows with the problem size (the dimension of the system matrices) and has not a physical foundation in the reality. Therefore, it is efficient to apply the previous numerical methods. Application of the Monte Carlo method in IFEA may be realized as follows:

\section{Generation of the random matrix (uniform distribu-} tion)

$$
\mathbf{X}_{\mathrm{MC}}=\left[\mathbf{x}_{1}, \ldots . . \mathbf{x}_{\mathrm{m}}\right],(\mathrm{m} \approx 5000 \div 100000)
$$

\section{Solution of}

$\boldsymbol{\lambda}_{j_{-} M C} \rightarrow\left[\mathbf{K}\left(\mathbf{x}_{j}\right)-\lambda_{j_{-} M C} \cdot \mathbf{M}\left(\mathbf{x}_{j}\right)\right] \cdot \mathbf{V}_{j}=\mathbf{0}$

for $i=1, \ldots, \mathrm{m}$.

\section{Infimum calculation of the $i$-th eigenvalue}

$\underline{\lambda_{i}}=\inf \left(i^{\text {th }}\right.$ row of $\left.\lambda_{\mathrm{COM} 2}\right)$

supremum calculation of the i-th eigenvalue

$\overline{\lambda_{i}}=\sup \left(i^{\text {th }}\right.$ row of $\left.\lambda_{\text {COM } 2}\right)$

In the case of COM1, the numerical approach implementation to IFEA is following:

infimum calculation

$\underline{\lambda} \rightarrow[\mathbf{K}(\underline{\mathbf{x}})-\underline{\lambda} \cdot \mathbf{M}(\underline{\mathbf{x}})] \cdot \underline{\mathbf{V}}=\mathbf{0}$,

- supremum calculation

$\bar{\lambda} \rightarrow[\mathbf{K}(\overline{\mathbf{x}})-\bar{\lambda} \cdot \mathbf{M}(\overline{\mathbf{x}})] \cdot \overline{\mathbf{V}}=\mathbf{0}$

COM1 doesn't give the correct results every time. We can obtain more proper results using COM2.

Its computational process for IFEA is:

1. Calculation of realizations matrix $\mathrm{X}_{2}$, i.e. $2^{\mathrm{n}}$ inf-sup combinations

$\mathbf{X}_{C O M 2}=\left[\mathbf{x}_{1}, \ldots . . \mathbf{x}_{m}\right],\left(\mathrm{m}=2^{\mathrm{n}}\right)$

$n$ - number of uncertain system parameters;

2.Solution of

$\boldsymbol{\lambda}_{j_{-} \text {СOM } 2} \rightarrow\left[\mathbf{K}\left(\mathbf{x}_{j}\right)-\boldsymbol{\lambda}_{j_{-} \text {СOM } 2} \cdot \mathbf{M}\left(\mathbf{x}_{j}\right)\right] \cdot \mathbf{V}_{j}=\mathbf{0}$

for $j=1, \ldots, \mathrm{m}$.

\section{Infimum calculation of the $i$-th eigenvalue}

$\underline{\lambda_{i}}=\inf \left(i^{\text {th }}\right.$ row of $\left.\lambda_{\text {COM } 2}\right)$

supremum calculation of the $i$-th eigenvalue

$\bar{\lambda}_{i}=\sup \left(i^{\text {th }}\right.$ row of $\left.\lambda_{\text {COM } 2}\right)$

Generally, the infimum or supremum are not found only in the boundary points (COM1, COM2) but also in the inner domain of the solution set (OPT). To find the inf-sup solution using the approach OPT means to solve the optimizing problem de- 
scribed as follows:

\section{ninfimum calculation of the $i$-th eigenvalue}

$$
\begin{array}{ll}
\frac{\lambda_{i}}{\left(\mathrm{x}_{O P T}\right) \rightarrow} & \lambda_{i} \\
{\left[\mathrm{~K}(\mathrm{x})-\lambda_{i} \cdot \mathrm{M}(\mathrm{x})\right] \cdot \mathrm{V}_{i}=0,}
\end{array}
$$

\section{nupremum calculation of the $i$-th eigenvalue}

$\bar{\lambda}_{i}\left(\mathrm{x}_{O P T}\right) \rightarrow$

$\lambda_{i} \quad$.

$\left[\mathrm{K}(\mathrm{x})-\lambda_{i} \cdot \mathrm{M}(\mathrm{x})\right] \cdot \mathrm{V}_{i}=0$.

It should be noted that it is possible to realize the searching process by a comparison optimizing method (e.g. Nelder-Mead simplex algorithm) or by using genetic algorithm as a robust tool of global optimization.

\section{Fuzzy Set Approach}

By Zadeh [5] was formulated the initial theory of fuzzy sets. A fuzzy set $\boldsymbol{x}$ is the set with boundaries that are not sharply defined. A function, called membership function (MSF), signifies the degree to which each member of a domain $\mathrm{X}$ belongs to the fuzzy set $\boldsymbol{x}[3,5,7]$. For a fuzzy variable $x \in\left\langle x_{1}, x_{2}\right\rangle$, (or $x \in \boldsymbol{x}$ ), the membership function is defined as $\mu(x)$. If $\mu(x)=1, x$ is definitely a member of the $\boldsymbol{x}$. If $\mu(x)=0, \mathrm{x}$ is definitely not a member of the $\boldsymbol{x}$. For every $x$ with $0<\mu(x)<1$, the membership is not certain. By fuzzy technique, the complete information about the uncertainties in the model can be included and one can demonstrate how these uncertainties are processed through the calculation procedure in MATLAB [8].
Numerical calculation of the fuzzy sets is usually realised using so called discrete fuzzy number. It means each fuzzy number is divided into $n$ interval numbers with $\alpha$-level MSF, where $\alpha \in<0,1>$. On each level is applied one of the presented numerical approaches applicable for interval analysis. The whole computational procedure is presented on Fig. 1. More details about implementation of this fuzzy computational technique into FEA as fuzzy finite element analysis (FFEA) inhere in the relevant literature [1]-[3], [7].

\section{PROBABILISTIC APPROACH}

Probabilistic approach is based on the transformation of random variables. Lets $\mathrm{X}=\left(X_{1}, \ldots, X_{\mathrm{n}}\right)^{T}$ be a $n$-dimensional random variable with joint probability distribution function $f_{\mathrm{x}}\left(x_{1}, \ldots, x_{\mathrm{n}}\right)$. We find joint probability distribution function $\mathrm{g}_{\mathrm{Y}}\left(y_{1}, \ldots, y_{\mathrm{n}}\right)$ of $n$-dimen-sional random variable $\mathbf{Y}=\left(Y_{1}, \ldots, Y_{\mathrm{n}}\right)^{T}$, where $Y_{j}=h_{j}\left(X_{1}, \ldots, X_{\mathrm{n}}\right), j=\mathbf{1}, \ldots, \mathrm{n} ; \mathbf{h}=\left(h_{1}, \ldots, h_{\mathrm{n}}\right)^{T}$ is $1: 1$ transformation of $X$ into $Y$.

Lets inverse function $\mathbf{h}^{-1}: \mathbf{X}=\mathbf{h}^{-1}(\mathbf{y})$ is diffe-rentiable and Jacobian of the transformation $J$,

$$
\begin{gathered}
J=\left|\begin{array}{ccc}
\frac{\partial h_{1}^{-1}}{\partial y_{1}} & \ldots & \frac{\partial h_{1}^{-1}}{\partial y_{n}} \\
\ldots & \ldots & \ldots \\
\frac{\partial h_{n}^{-1}}{\partial y_{1}} & \ldots & \frac{\partial h_{n}^{-1}}{\partial y_{n}}
\end{array}\right| \quad \frac{1}{J}=\left|\begin{array}{ccc}
\frac{\partial h_{1}}{\partial x_{1}} & \ldots & \frac{\partial h_{1}}{\partial x_{n}} \\
\ldots & \ldots & \ldots \\
\frac{\partial h_{n}}{\partial x_{1}} & \ldots & \frac{\partial h_{n}}{\partial x_{n}}
\end{array}\right| \\
J \neq 0
\end{gathered}
$$

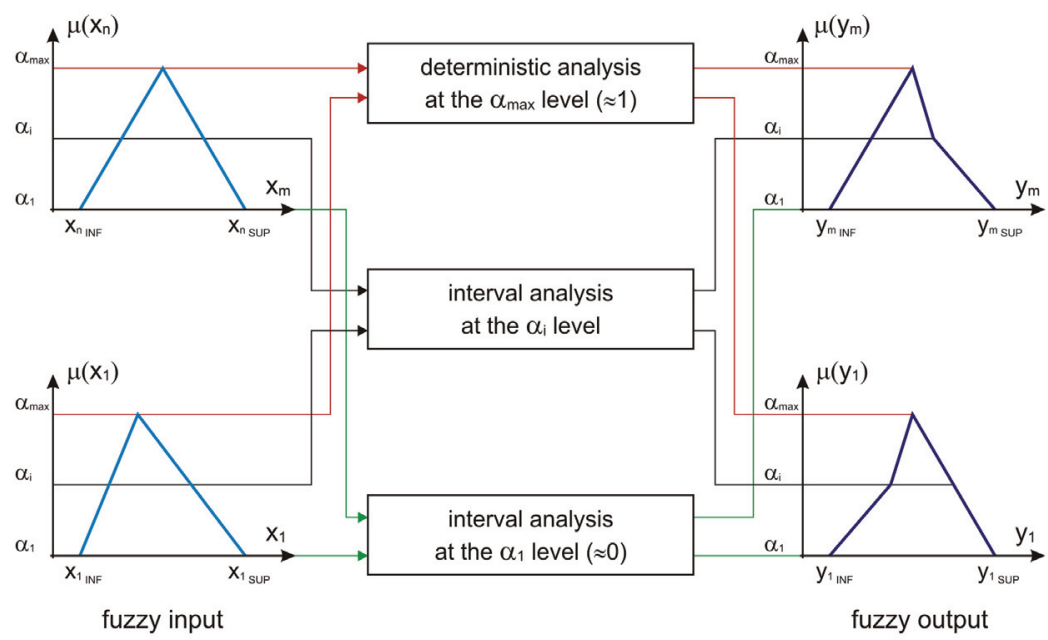

Fig. $1 \alpha$-level strategy with $n$-input levels and $m$-output levels, for a function of triangular fuzzy parameters 
The joint probability distribution function $\mathrm{g}_{\mathrm{Y}}$

$$
\begin{aligned}
& g_{\mathbf{Y}}(\mathbf{y})=g_{\mathbf{Y}}\left(y_{1}, \ldots, y_{n}\right)= \\
& =f_{\mathbf{X}}\left[h_{1}^{-1}\left(y_{1}, \ldots, y_{n}\right), \ldots, h_{n}^{-1}\left(y_{1}, \ldots, y_{n}\right)\right] \cdot J= \\
& =f_{\mathbf{X}}\left[\mathbf{h}^{-1}(\mathbf{y})\right] \cdot J
\end{aligned}
$$

Probability density function $f_{x}(x)$ is for uniform distribution of random variable $X$ on the interval $\left(a_{1,}\right.$ $\left.a_{3}\right), X \sim$ Unif $\left(a_{1}, a_{3}\right)$, Fig. 2.

$$
\begin{array}{rl}
f_{X}(x)=\left\{\begin{aligned}
0, & x \notin\left\langle a_{1}, a_{3}\right\rangle \\
\frac{1}{a_{3}-a_{1}}, & x \in\left\langle a_{1}, a_{3}\right\rangle
\end{aligned}\right. \\
f_{\mathbf{x}}(x) & \\
\hline v & v \\
\hline a_{1} & v=1 /\left(a_{3}-a_{1}\right)
\end{array}
$$

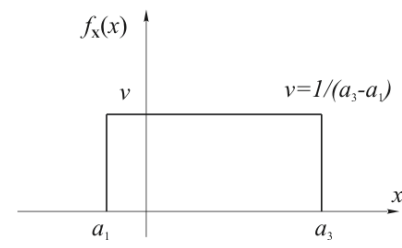

Fig. 2 Uniform distribution, Unif $\left(a_{1}, a_{3}\right)$

Probability density function $f_{x}(x)$ is for triangular distribution of random variable $X$ on the interval $\left(a_{1}, a_{3}\right), X \sim \operatorname{Tri}\left(a_{1}, a_{2}, a_{3}\right)$, Fig. 3.

$$
f_{X}(x)=\left\{\begin{array}{cc}
0, & x \notin\left(a_{1}, a_{3}\right\rangle \\
\frac{2\left(x-a_{1}\right)}{\left(a_{3}-a_{1}\right)\left(a_{2}-a_{1}\right)}, & x \in\left\langle a_{1}, a_{2}\right\rangle \\
\frac{2\left(a_{3}-x\right)}{\left(a_{3}-a_{1}\right)\left(a_{3}-a_{2}\right)}, & x \in\left(a_{2}, a_{3}\right\rangle
\end{array}\right.
$$

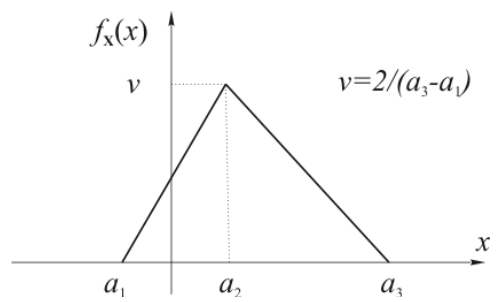

Fig. 3 Triangular distribution, Tri $\left(a_{1}, a_{2}, a_{3}\right)$

Calculation of the function $\mathrm{g}_{\mathrm{Y}}$ can be very difficult. The increasing of dimension of independent random variable $\mathbf{X}$ means generally increasing of difficult ant time consuming too. We will use Monte Carlo method to create the random samples of variable $\mathbf{Y}$. We can use next process by determina- tion these random samples of $\mathbf{Y}$ :

1.Generation of input vector $\mathrm{X}=\left(X_{1}, \ldots, X_{\mathrm{n}}\right)^{T}$ by using random number generator,

2.Obtain of output vector $\mathrm{Y}=\left(Y_{1}, \ldots, Y_{\mathrm{n}}\right)^{T}$ by using $\mathbf{h}=\left(h_{1}, \ldots, h_{\mathrm{n}}\right)^{T}$ transformation,

3.Saving file with Y or processing this vector immediately,

4. repeat step from $1^{\text {st }}$ to $3^{\text {rd }}$, until the required number of vectors $\mathrm{Y}$ is fulfilled.

The processing of output vectors is based on aims of analysis, for example a histogram, an estimation of parameters of distribution function $f_{\mathrm{x}}\left(x_{1}, \ldots, x_{\mathrm{n}}\right)$ sample mean, sample median, sample standard deviation etc.

The comparison of the shape of histogram with interval or fuzy set estimation is used in the next parts. Probability interpretation of output vector $\mathbf{Y}$ is often used.

A probability $P$ :

$P\left\{{ }_{1} \leq Y_{1}<b_{1}, \ldots \leq Y_{n}<b_{n}\right\}=$

$=\int_{a_{1}}^{b_{1}} \ldots \int_{a_{n}}^{b_{n}} g_{\mathrm{Y}}\left(y_{1}, \ldots, y_{n}\right) d y_{1} \ldots d y_{2}=$

$=\int_{a_{1-}}^{b_{1+}} \ldots \int_{a_{n-}}^{b_{n+}} I_{\mathrm{Y}}\left(y_{1}, \ldots, y_{n}\right) g_{\mathrm{Y}}\left(y_{1}, \ldots, y_{n}\right) d y_{1} \ldots d y_{2}$.

Where $\mathrm{a}_{i-} \leq \mathrm{a}_{i}<\mathrm{b}_{i} \leq \mathrm{b}_{i+},\left(\mathrm{a}_{i-\mathrm{s}}, \mathrm{b}_{i+}\right)$ is interval for values $Y_{i,} i \in\{1, \ldots, n\}$, after transformation $\mathbf{h}$,

$I_{\mathbf{Y}}\left(y_{1}, \ldots, y_{n}\right)=I_{\mathbf{Y}}(\mathbf{y})=$

$= \begin{cases}1, & \forall i \in\{1, \ldots n\}, y_{i} \in\left\langle\mathrm{a}_{i}, b_{i}\right) ; \\ 0, & \exists i \in\{1, \ldots n\}, y_{i} \notin\left\langle\mathrm{a}_{i}, b_{i}\right),\end{cases}$

where $I_{\mathrm{Y}}(\mathbf{y})$ is indicator function.

By using indicator function we can modify $3^{\text {rd }}$ step. For generated values of vector $\mathbf{Y}$ we determine indicator function. If $N$ is the required number of vectors, than point estimation of probability $\hat{P}_{p o \text { int }}$ :

$\hat{P}_{\text {point }}=\frac{1}{N} \sum_{i=1}^{N} I_{\mathbf{Y}}\left(\mathbf{y}_{i}\right)$

An interval estimation $\hat{P}_{\text {int }}$ of probability:

$\left(\hat{P}_{\text {point }}-\operatorname{Er}(N), \quad \hat{P}_{\text {point }}+\operatorname{Er}(N)\right)$

where: 


$$
\begin{aligned}
& \left|P-\hat{P}_{\text {point }}\right|<x_{\gamma} \sqrt{\frac{\operatorname{var}\left[I_{\mathbf{Y}}(\mathbf{y})\right]}{N}}=\operatorname{Er}(N) \\
& \gamma \in(0,1), \gamma=\Phi_{0}\left(x_{\gamma}\right)=\frac{2}{\sqrt{2 \pi}} \int_{0}^{x_{\gamma}} e^{-\frac{t^{2}}{2}} d t
\end{aligned}
$$

a variance $\left[I_{\mathbf{Y}}(\mathbf{y})\right]$ we can use estimation [9]

$$
\begin{aligned}
& \operatorname{vâr}\left[I_{\mathbf{Y}}(\mathbf{y})\right]= \\
& =\frac{1}{N-1} \sum_{i=1}^{N} I_{\mathbf{Y}}^{2}\left(\mathbf{y}_{i}\right)-\frac{1}{N(N-1)}\left(\sum_{i=1}^{N} I_{\mathbf{Y}}\left(\mathbf{y}_{i}\right)\right)^{2}
\end{aligned}
$$

By using binomial properties of $I_{\mathrm{Y}}(\mathbf{y})$ and for condition [10]

$$
N \hat{P}_{\text {point }}\left(1-\hat{P}_{\text {point }}\right)>9
$$

the interval estimation is:

$$
\left(\begin{array}{c}
\hat{P}_{\text {point }}-x_{\gamma} \sqrt{\frac{\hat{P}_{\text {point }}\left(1-\hat{P}_{\text {point }}\right)}{N},} \\
\hat{P}_{\text {point }}+x_{\gamma} \sqrt{\frac{\hat{P}_{\text {point }}\left(1-\hat{P}_{\text {point }}\right)}{N}}
\end{array}\right)
$$

We can estimate $\mathbf{Y}$ in probability sense properties.

\section{NUMERICAL EXAMPLE}

Vehicle dynamic models are often characterized by uncertain system parameters. Main goal of this example will be to explain difference between three different approaches to the analysis the influence of the uncertain parameters on the natural frequencies case of the model. Let's consider the 7-DOFs model of the agricultural tractor (Fig. 4) [4]. All input parameters without stiffness will be constants. The stiffnesses are modeled as follows

\section{Deterministic (constant values) [N/m]:}

$k_{1}=3.10^{5}, k_{2}=2.10^{4}, k_{3}=1.10^{5}, k_{4}=2.10^{5}$.

\section{Interval numbers:}

$$
\begin{aligned}
& k_{1}=\left\langle 2,9399.10^{5}, 3,0601.10^{5}\right\rangle \\
& k_{2}=\left\langle 1,9599.10^{4}, 2,0401.10^{4}\right\rangle \\
& k_{3}=\left\langle 0,9799.10^{5}, 1,0201.10^{5}\right\rangle \\
& k_{4}=\left\langle 1,9599.10^{5}, 2,0401.10^{5}\right\rangle
\end{aligned}
$$

\section{Triangular fuzzy numbers:}

$k_{1}=\left\langle 2,9399.10^{5}, 3.10^{5}, 3,0601.10^{5}\right\rangle$

$k_{2}=\left\langle 1,9599.10^{4}, 2.10^{4}, 2,0401.10^{4}\right\rangle$

$k_{3}=\left\langle 0,9799.10^{5}, 1.10^{5}, 1,0201.10^{5}\right\rangle$

$k_{4}=\left\langle 1,9599.10^{5}, 2.10^{5}, 2,0401.10^{5}\right\rangle$

\section{Random variables:}

- Uniform distribution Unif (a, b):

$k_{1} \sim$ Unif $\left(2,9399.10^{5}, 3,0601.10^{5}\right)$,

$k_{2} \sim \operatorname{Unif}\left(1,9599.10^{4}, 2,0401.10^{4}\right)$

$k_{3} \sim \operatorname{Unif}\left(0,9799.10^{5}, 1,0201.10^{5}\right)$,

$k_{4} \sim \operatorname{Unif}\left(1,9599.10^{5}, 2,0401.10^{5}\right)$;

- Triangular distribution Tri (a, c, b):

$k_{1} \sim \operatorname{Tri}\left(2,9399.10^{5}, 3.105,3,0601.10^{5}\right)$,

$k_{2} \sim \operatorname{Tri}\left(1,9599.104,2.10^{4}, 2,0401.10^{4}\right)$,

$k_{3} \sim \operatorname{Tri}\left(0,9799.105,10^{5}, 1,0201.10^{5}\right)$,

$k_{4} \sim \operatorname{Tri}\left(1,9599.105,2.10^{5}, 2,0401.10^{5}\right)$.

The first three natural frequencies (eigenfre-quencies) are presented in Tab. 1 (for models

1-3). Result is presented in Tab. 2 for model number 4. The first and third eigenfrequencies for triangular density functions and fuzzy approach are shown on Fig. 5a,b. First and third eigenfrequencies for uniform density functions are shown on Fig. 6a,b.

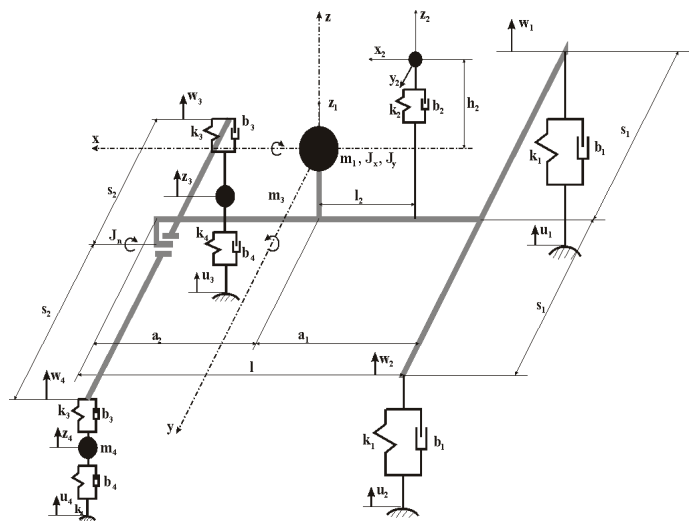

Fig. 4 The dynamic model of agricultural tractor

A soft histogram shift in left is caused by option of containers. "The teeth" can be decreasing by increasing number of samples (105 samples). The probability density function is approximately triangular/rectangle for triangular/uniform proba-bility density function of input variables.

On the figure number $5 a, b$ is obvious difference between fuzzy and probability approach results. 


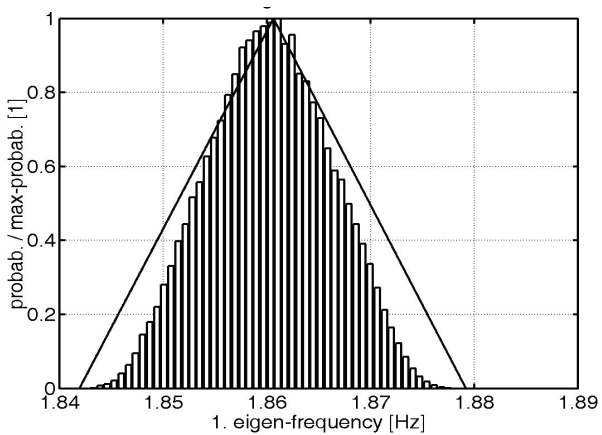

Fig. 5a Fuzzy and triangular distribution

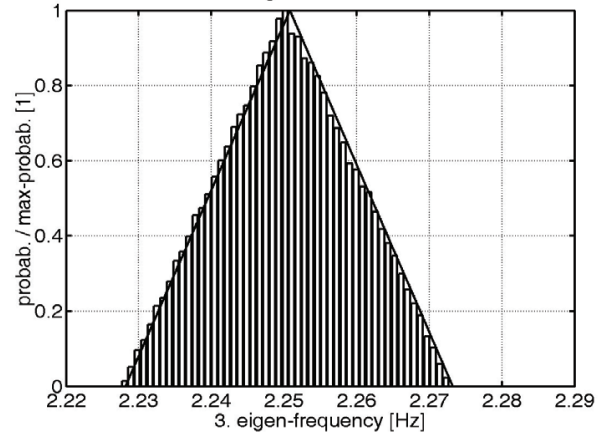

Fig. 5b Fuzzy and triangular distribution

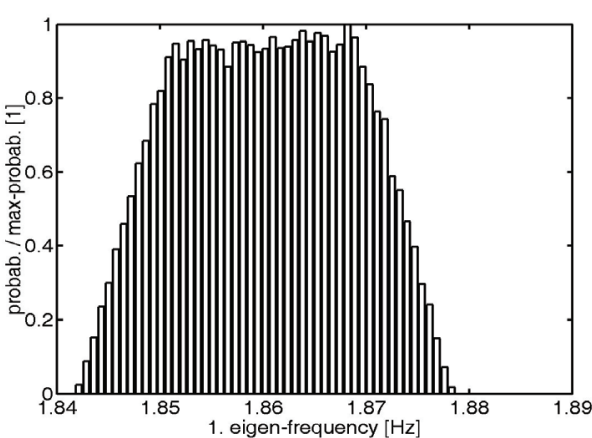

Fig. 6a Uniform distribution

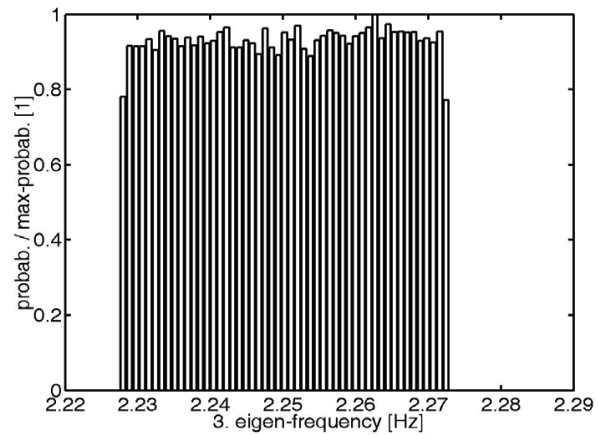

Fig. 6b Uniform distribution

\begin{tabular}{|l|l|l|l|}
\hline & \multicolumn{1}{c}{ Constant model } & \multicolumn{1}{c|}{ Interval model } & \multicolumn{1}{c|}{ Fuzzy model } \\
\hline $\mathrm{f}_{1}$ & 1,8606 & $<1,8419 ; 1,8791>$ & $<1,8419 ; 1,8606 ; 1,8792>$ \\
\hline $\mathrm{f}_{2}$ & 2,1126 & $<1,8419 ; 1,8791>$ & $<1,8419 ; 1,8606 ; 1,8792>$ \\
\hline $\mathrm{f}_{3}$ & 2,2508 & $<1,8419 ; 1,8791>$ & $<1,8419 ; 1,8606 ; 1,8792>$ \\
\hline
\end{tabular}

Tab. 1 The first three eigenfrequencies $[\mathrm{Hz}]$ are presented for constant, interval and fuzzy model

\begin{tabular}{|c|c|c|c|c|}
\hline \multirow[t]{2}{*}{ Natural Frequency } & \multirow[t]{2}{*}{ Mean value } & \multicolumn{3}{|c|}{ Confidence interval } \\
\hline & & $99 \%$ & $95 \%$ & $90 \%$ \\
\hline 1. & 1,86057 & $(1,8486 ; 1,8726)$ & $(1,8513 ; 1,8699)$ & $(1,8527 ; 1,8684)$ \\
\hline 2. & 2,11261 & $(2,1000 ; 2,1252)$ & $(2,1029 ; 2,1223)$ & $(2,1044 ; 2,1208)$ \\
\hline 3. & 2,25076 & $(2,2320 ; 2,2694)$ & $(2,2362 ; 2,2652)$ & $(2,2385 ; 2,2631)$ \\
\hline
\end{tabular}

Tab. 2 The first three eigenfrequencies are presented for triangular probability density function

There is visible difference in the range of interval for interpretations fuzzy versus probability too.

If we will obtain the results for interval approach and will extend interpretation of results in probability sense (interval number $\approx$ random variable with uniform distribution) then result have no uniform distribution.

\section{CONCLUSION}

The paper deals with the possibilities of the interval, fuzzy a probability approaches in fundamen- tal problems of the mechanics as are a modal and spectral FE analysis, mechanism kinematic analysis etc.. The presented comparison study of interval numbers, fuzzy set and probabilistic approaches from point of view results interpretation. Each of these accesses have itself advantage. Study is warned against risk of miss-interpretation of results, in the case interpretation of interval numbers as a realization of uniform distributed random variable, or in the case interpretation triangular fuzzy number as a realization random variable with tri- 
angular probability density function.

The use of the presented numerical approaches provides a new possibility of the quality and reliability appraisal of analyzed objects. Due to this numerical approach, we can analyze mechanical, technological, service and economic properties of the investigated structures more authentically.

\section{ACKNOWLEDGEMENT}

This work has been supported by VEGA grant No. 1/0125/09 and VEGA grant No. 1/0727/10.

\section{REFERENCES}

[1] Elishakoff I., Duan D., Application of Mathematical Theory of Interval Analysis to Uncertain Vibrations, Proc. of NOISE-CON'94, Ft. Lauderdale, Florida, 1994, p. 519-524

[2] Forssén P., Interval methods I, http://www. tdb.uu.se/kurs/optim-mn1/ht01/lectures/ lec14_2.pdf

[3] Chen S.H., Yang X.W., Interval Finite Element Method for Beam Structures, Finite Elem. Anal. Des., 34, 2000, pp. 75-88

[4] Kudlička J., Dynamické skúmanie polno hospodárskeho traktora UR IV, Strojnícky časopis, 45, 1994, č.6, str. 543-549

[5] Kulpa Z., Pownuk A., Skalna I., Analysis of Linear Mechanical Structures with Uncertainties by Means of Interval Methods, Computer Assisted Mechanics and Engineering Sciences, Vol. 5, 1998, p. 443-477.

[6] Moore R.E., Interval Analysis, Prentice Hall, Englewood Cliffs, New Jersey, 1966.

[7] Neumaier A., Interval Methods for Systems of Equations, Cambridge University Press, Cambridge, 1990.

[8] Zhang H., Muhanna R.L., Finite Element Analysis for Structures with Interval Parameters, Proc. 9th ASCE Joint Sp. Conf. on Probab. Mech. and Struct. Reliability, Albuquerque, New Mexico, USA, 2004

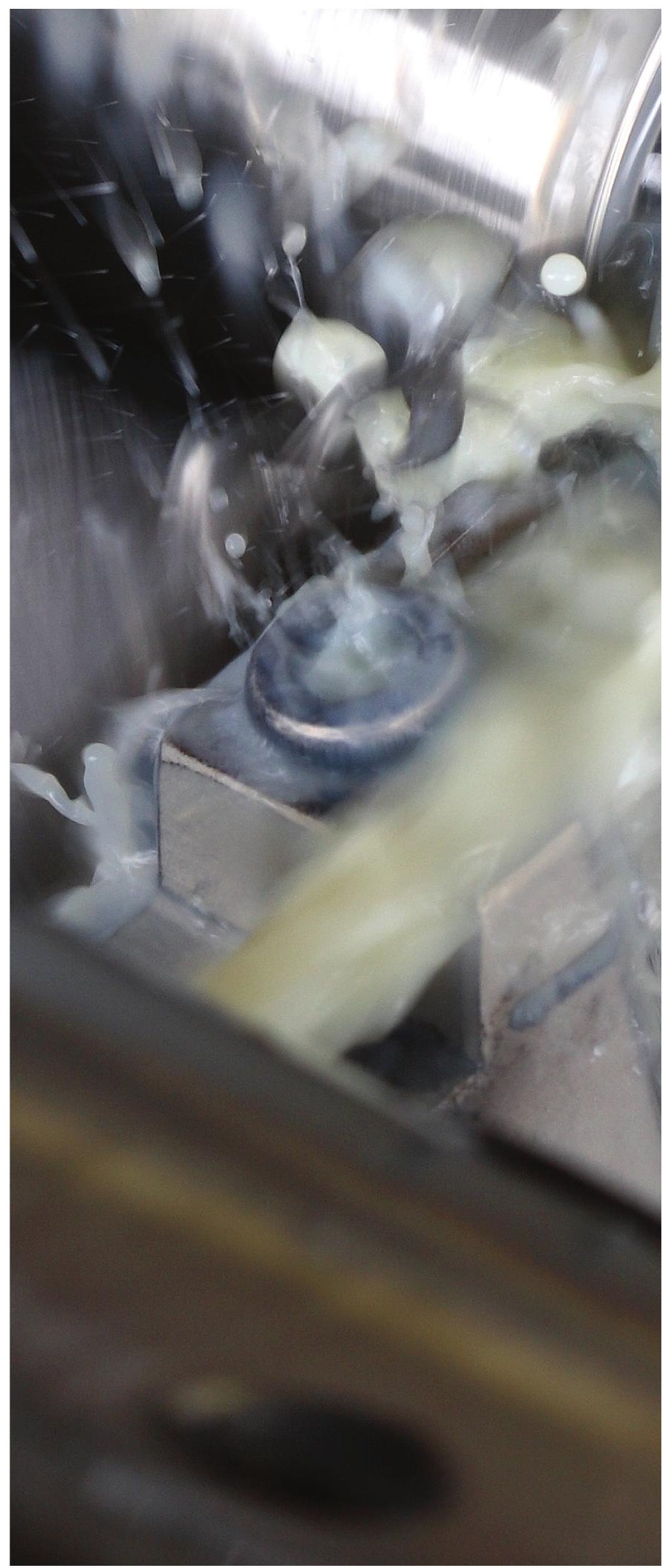




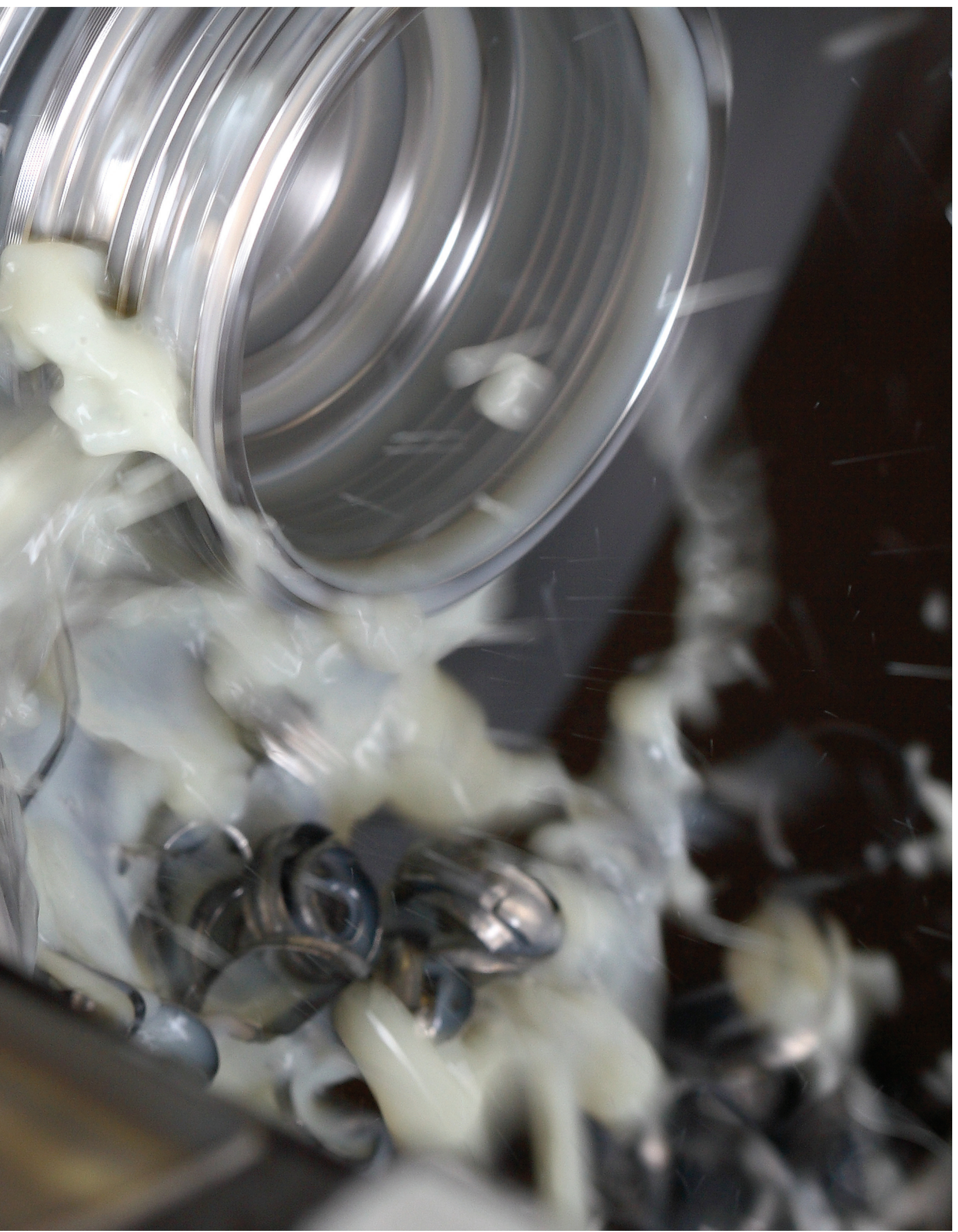

